\title{
A Compact Photonic Horizontal Spot-Size Converter Realized in Silicon-on-Insulator
}

\author{
Bert Luyssaert, Student Member, IEEE, Peter Vandersteegen, Student Member, IEEE, \\ Dirk Taillaert, Student Member, IEEE, Pieter Dumon, Student Member, IEEE, Wim Bogaerts, Student Member, IEEE, \\ Peter Bienstman, Member, IEEE, Dries Van Thourhout, Member, IEEE, Vincent Wiaux, Stephan Beckx, and \\ Roel Baets, Senior Member, IEEE
}

\begin{abstract}
We present a compact planar coupler connecting two optical waveguides with highly different widths. The coupler consists of various nonperiodic waveguide sections, whose dimensions are determined using a genetic optimization algorithm. Efficiencies that exceed those of the more conventional designs with similar lengths, like gradual linear tapers, were obtained in silicon-on-insulator using 248-nm-deep ultraviolet lithography.
\end{abstract}

Index Terms-Compact coupler, high index contrast, nonadiabatic taper, optical coupler.

\section{INTRODUCTION}

D UE TO its excellent light confinement and due to the maturity of silicon processing technology, silicon-on-insulator (SOI) has become an important material for research on passive photonic components. Various functional basic components have already been demonstrated in this material system, such as low-loss photonic wires and bends [1], low-loss photonic crystal waveguides [2], and high- $Q$ cavities to couple light between different waveguides [3].

Within a more complex photonic circuit, waveguides with different widths have to be used together. This will occur if photonic wires are applied where monomodality is important but broader waveguides for connections that need to be very low-loss or for coupling to an optical fiber.

To couple optical waveguides with different cross sections and different modal sizes, smooth linear or parabolic tapers can be used [4]. However, in order to become adiabatic or lossless, these tapers need to be sufficiently long. In complex photonic integrated circuits, every component should occupy a footprint that is as small as possible, to reduce material, processing, and packaging costs. Therefore, shorter innovative coupler designs are needed. In [5], an improvement in coupling efficiency of a

\footnotetext{
Manuscript received July 6, 2004; revised September 3, 2004. This work was supported in part by the IST-PICCO Project supported by the European Union and in the context of the Belgian IAP PHOTON Network. The work of B. Luyssaert, P. Dumon, and W. Bogaerts was supported by the Flemish Institute for the Industrial Advancement of Scientific and Technological Research (IWT) under a specialization grant. The work of P. Bienstman was supported by the Flemish Fund for Scientific Research (FWO-Vlaanderen) through a postdoctoral fellowship.

B. Luyssaert, P. Vandersteegen, D. Taillaert, P. Dumon, W. Bogaerts, P. Bienstman, D. Van Thourhout, and R. Baets are with the Photonics Research Group, Department of Information Technology (INTEC), Ghent University-IMEC, Gent B-9000, Belgium (e-mail: Bert.Luyssaert@ intec.UGent.be).

V. Wiaux and S. Beckx are with the Silicon Processing Technology Division, IMEC vzw, Leuven B-3001, Belgium (e-mail: vincent.wiaux@imec.be).

Digital Object Identifier 10.1109/LPT.2004.838655
}

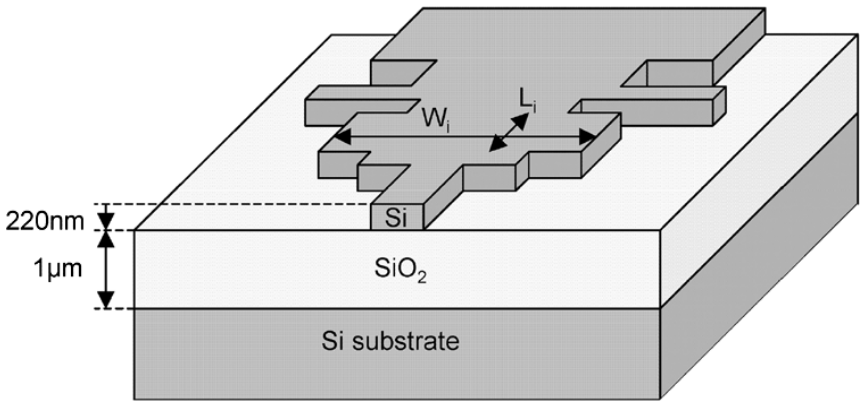

Fig. 1. Schematic presentation of the component with the layer structure indicated.

fiber-to-silica ridge waveguide was shown, using instead of a direct butt-coupling scheme a short planar coupler fabricated in a low index material $\left(\mathrm{SiO}_{2}\right)$.

In this letter, we present a device based on the same principle (see Fig. 1), but now realized in SOI, a high refractive index contrast material system. Instead of an optical fiber and a silica ridge waveguide, two planar waveguides with the same vertical layer structure but different widths, are coupled. Due to the higher refractive index contrast other mechanisms play a role than in [5] and other simulations tools must, therefore, be applied.

\section{DESIGN}

As a high refractive index contrast can easily lead to reflection, a bidirectional and nonparaxial simulation method has to be used to calculate these structures. For reasons of speed and easy modal analysis an eigenmode expansion (EME) frequency domain method, with a perfectly matched layer as absorbing boundary condition, was chosen above a finite-difference time-domain (FDTD) method.

A first optimization was done in a two-dimensional (2-D) EME simulation, using CAMFR [6], after an effective index transformation on our SOI layer structure $(0.22-\mu \mathrm{m} \mathrm{Si} / 1.0-\mu \mathrm{m}$ silica/Si-substrate), resulting in an effective core index of 2.83 for a wavelength of $1.56 \mu \mathrm{m}$. For the transverse-magnetic mode, the dominant electric field component is parallel to the SOI layer structure. The structure to be optimized is shown in Fig. 1; the widths of the input and output waveguides are 10 and $0.56 \mu \mathrm{m}$. Between two straight waveguides with a different width, a number of waveguide segments with random width and length are placed. $N$ waveguide segments lead to $2 N$ variables $\left[L_{1}, \ldots, L_{N}, W_{1}, \ldots, W_{N}\right]$, which are the input parameters to a genetic optimization algorithm. Certain optimized sets 


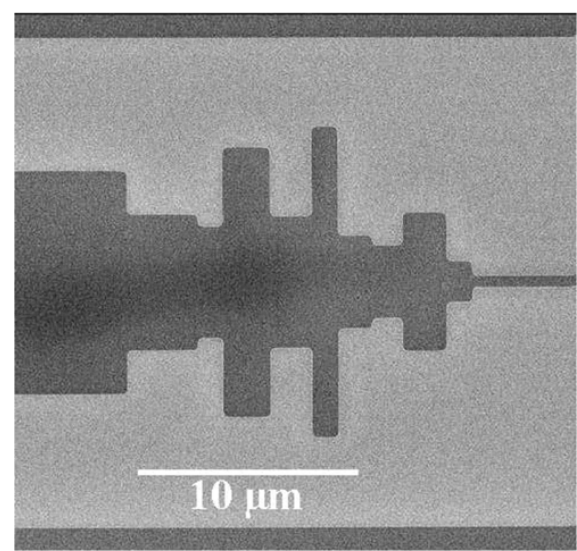

Fig. 2. SEM picture of the component realized in SOI. The input and output waveguide have a width of, respectively, 10.0 and $0.56 \mu \mathrm{m}$. The total length of the coupler is $15.4 \mu \mathrm{m}$. Light gray corresponds to areas where the top silicon layer has been etched away and the underlying silica layer is revealed.

of these parameters lead to a good ground mode intensity transmission between the input and output waveguide.

Working with only ten sections each with a length of around $1.5 \mu \mathrm{m}$, the optimized set of parameters leads to a power transmission efficiency of $78 \%$ in a 2-D calculation. By using more sections while keeping the total length constant, much better transmissions can be achieved, up to $95 \%$, at the cost of longer optimization times, and more importantly, smaller features within the structure. The coupler with $78 \%$ transmission efficiency is well within the boundaries of the technological capability of deep ultraviolet (UV) lithography (in terms of the smallest feature size, approximately $150 \mathrm{~nm}$ ), and has, therefore, been chosen as proof-of-principle. Using this optimized set, the resulting structure is then rigorously calculated in three dimensions (3-D), also using EME. A further optimization in 3-D follows, using the commercial optimizer KALLISTOS [7] in local optimization mode, meaning that an optimum, nearby in the multidimensional parameter space, is identified. This extra optimization step slightly improves the transmission from $71 \%$ to $72 \%$, for a coupler consisting of ten sections and with a total length of only $15.4 \mu \mathrm{m}$.

We have also tried to build a simplified model so as to understand the physics of the component, with the hope to gain insight and to derive analytical design rules. All these attempts, however, have failed so far.

\section{REALIZATION}

We realized the couplers in SOI using 248-nm-deep UV lithography. For fabrication details, we refer to [8], but contrasting with earlier experiments only the top silicon layer was etched, resulting in smaller sidewall roughness and, therefore, less scattering loss. Also, the silica cladding layer had a thickness of $1 \mu \mathrm{m}$ to minimize substrate losses. A scanning electron microscope (SEM) picture of a spot-size converter is shown in Fig. 2.

To provide coupling between standard silica fiber (9- $\mu \mathrm{m}$ core) and broad planar SOI waveguides, vertical fiber couplers [9], based on a second-order grating, were included in the design. An additional etching step, only $45 \mathrm{~nm}$ deep, applied before

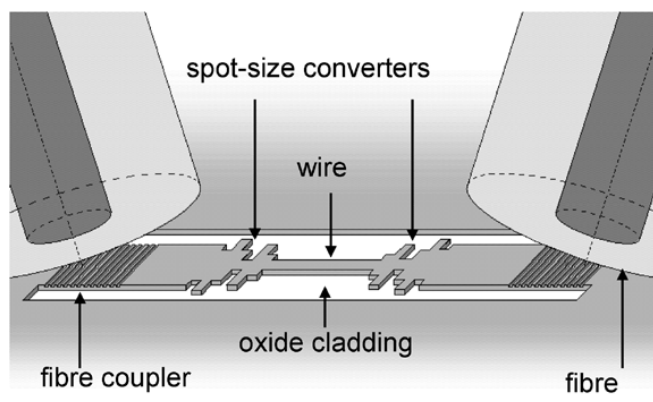

Fig. 3. Scheme of a typical structure and of the measurement setup, including the two vertical fiber couplers. Different elements are not necessarily shown on the same scale.

the waveguide etch, is needed to fabricate these fiber couplers. The grating period is chosen to optimize transmission around $1550 \mathrm{~nm}$ and to couple to a fiber with an inclination of $10^{\circ}$ away from the normal to the surface. This nonvertical inclination facilitates the measurement setup and, by breaking the symmetry, avoids reflection at the coupler grating back into the broad SOI waveguide. Using these vertical fiber couplers, optical quality facets, which are difficult to cleave in SOI and need an additional substrate thinning step, become unnecessary. This improves the yield and the speed of the entire fabrication and characterization process.

\section{Characterization}

Vertical fiber couplers for butt-coupled fibers based on a short one-dimensional grating have been used to characterize the devices [9]. A typical structure consists of a central 100- $\mu \mathrm{m}$-long SOI wire waveguide $(0.56 \mu \mathrm{m}$ wide and monomodal $)$ on both sides coupled to a broad SOI waveguide ( $10.0 \mu \mathrm{m}$ wide) using a compact spot-size converter. These broad waveguides extend on both sides to nearly the edge of the chip where the vertical fiber couplers are located, exactly $7 \mathrm{~mm}$ apart (see Fig. 3). Dedicated reference structures have a similar design and an identical fiber coupler spacing, but instead of compact spot-size converters, linear tapers ( $2.5 \mathrm{~mm}$ long) connect the central $100-\mu \mathrm{m}$-long wire and the broad waveguides. Having this length, the linear tapers fulfill the adiabatic condition and can be considered lossless, except for the propagation losses also found in straight waveguides. These losses amount to $2.4 \pm 1.6 \mathrm{~dB} / \mathrm{cm}$ for wires with a width of $0.5 \mu \mathrm{m}$ and less than $1.0 \mathrm{~dB} / \mathrm{cm}$ for waveguides with a width of $10.0 \mu \mathrm{m}$.

Fig. 4 shows the output spectra, measured fiber-to-fiber of both a reference and a compact coupler structure. To eliminate waveguide and incoupling losses and to calculate the efficiency, the spectrum of the coupler is divided by the spectrum of the reference structure, as can be seen in Fig. 5. Only the part indicated in thick line is of importance because at both ends of the spectrum, the error grows rapidly due to the low power values and the division of two curves with a steep slope. Depending on the exact positions of the two fibers in the setup, the Gaussian transmission curve of the reference structure can be slightly shifted to lower or higher wavelengths. However small, this shift has a major repercussion on the efficiency curve in the slope region. 


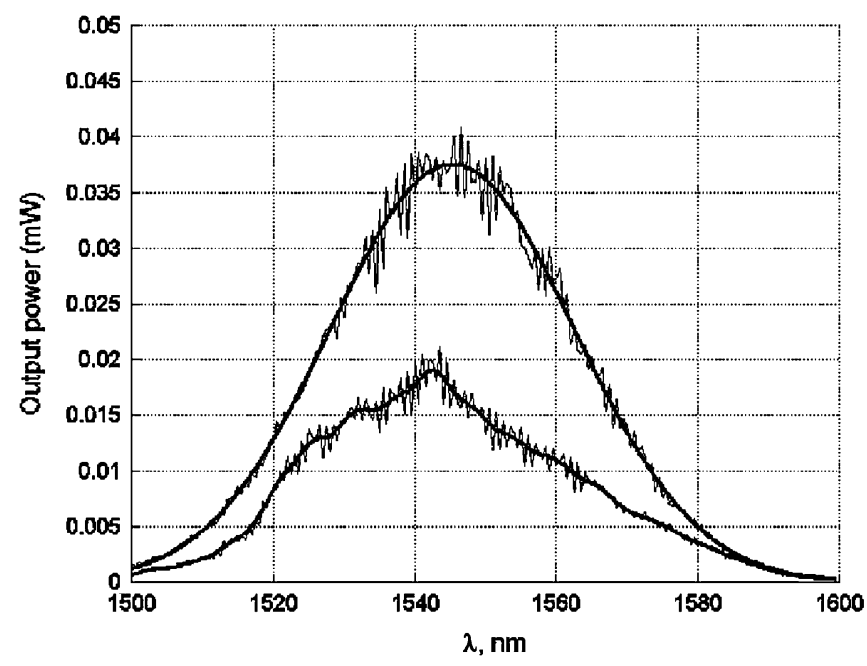

Fig. 4. Measured spectra of a reference structure and a compact spot-size converter. The upper curves show the measured output power of a reference structure and the best fitting Gaussian. The lower curves correspond to a pair of compact spot-size converters and a spectral average of these points.

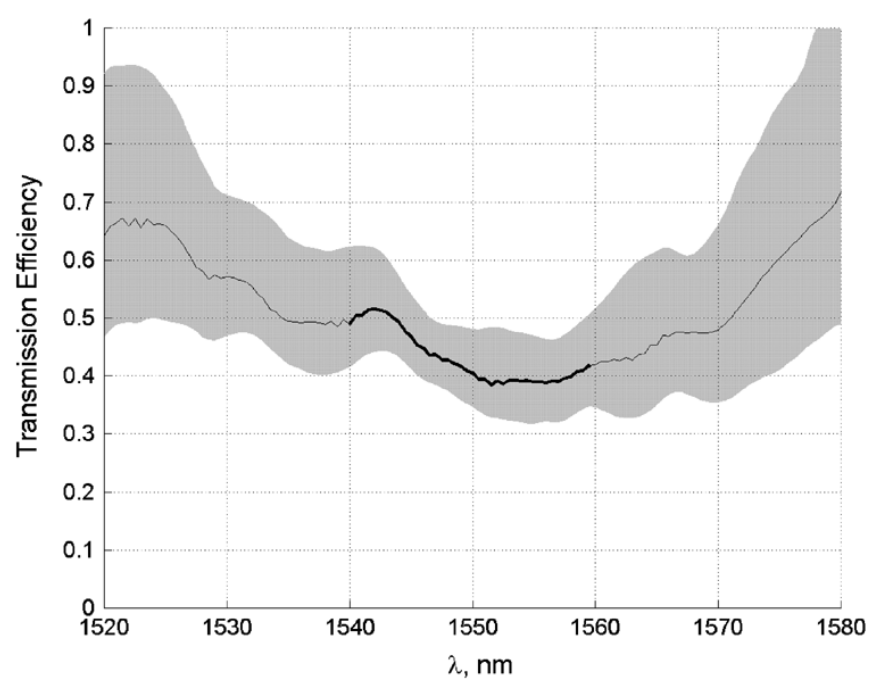

Fig. 5. Efficiency spectrum obtained by dividing the averaged compact coupler spectrum by the Gaussian fit to the reference spectrum. In the region of low error, the efficiency spectrum is indicated using a thick line.

In the region of importance, the efficiency is between $40 \%$ and $52 \%$. As this figure includes two couplers, a single spot-size converter has an efficiency between $63 \%$ and $72 \%$, coming very close to the calculated maximum value for this particular structure. Measurements on similar structures but with the compact spot-size converters replaced with short linear tapers and normalized in the same way, reveal a maximum efficiency of $40 \%$ for a single linear taper of $12.5 \mu \mathrm{m}$ and $59 \%$ for a linear taper of $25 \mu \mathrm{m}$. This clearly demonstrates that the compact coupler performs better than a significantly longer linear taper.

\section{CONCLUSION}

A compact spot-size converter consisting of several nonperiodic waveguide segments has for the first time been studied in a high refractive index contrast material to couple two different planar waveguides. After a 2-D optimization using a genetic optimization algorithm and a further local optimization in 3-D, both using EME, a design with a maximum theoretical efficiency of $72 \%$ has been found. The design has a total length of only $15.4 \mu \mathrm{m}$ and couples a waveguide with a width of $0.56 \mu \mathrm{m}$ to a waveguide with a width of $10 \mu \mathrm{m}$. This converter has been realized in SOI using deep UV lithography and first measurements indicate an efficiency between $63 \%$ and $72 \%$, outperforming linear tapers of similar and longer length. These results prove that other coupler designs than conventional adiabatic ones are useful components in future photonic integrated circuits.

\section{ACKNOWLEDGMENT}

The authors would also like to thank D. Vangoidsenhoven for the wafer exposures, R. de Ruyter and J. Mees for their work on the mask design, and J. Wouters for the extensive work on the etch development. Also many thanks to the people of P-Line for the processing.

\section{REFERENCES}

[1] Y. A. Vlasov and S. J. McNab, "Losses in single-mode silicon-on-insulator strip waveguides and bends," Opt. Expresss, vol. 12, no. 8, pp. 1622-1631, 2004.

[2] M. Notomi, A. Shinya, S. Mitsugi, E. Kuramochi, and H. Y. Ryu, "Waveguides, resonators and their coupled elements in photonic crystal slabs," Opt. Express, vol. 12, no. 8, pp. 1551-1561, 2004.

[3] H. Takano, Y. Akahane, T. Asano, and S. Noda, "In-plane-type channel drop filter in two-dimensional photonic crystal slabs," Appl. Phys. Lett., vol. 84, no. 13, pp. 2226-2228, 2004.

[4] C. K. Burns, A. F. Milton, and A. B. Lee, "Optical-waveguide parabolic coupling horns," Appl. Phys. Lett., vol. 30, no. 1, pp. 28-30, 1977.

[5] M. M. Spuhler, B. J. Offrein, G. L. Bona, R. Germann, I. Masserek, and D. Erni, "A very short planar silica spot-size converter using a nonperiodic segmented waveguide," J. Lightw. Technol., vol. 16, no. 9, pp. 1680-1685, Sep. 1998.

[6] P. Bienstman and R. Baets, "Optical modeling of photonic crystals and VCSEL's using eigenmode expansion and perfectly matched layers,' Opt. Quant. Elect., vol. 33, no. 4-5, pp. 327-341, 2001.

[7] T. Felici and H. W. Engl, "On shape optimization of optical waveguides using inverse problem techniques," Inverse Problems, vol. 17, pp. 1141-1162, 2001.

[8] W. Bogaerts, V. Wiaux, P. Dumon, D. Taillaert, J. Wouters, S. Beckx, J. Van Campenhout, B. Luyssaert, D. Van Thourhout, and R. Baets, "Large-scale production techniques for photonic nanostructures," Proc. SPIE, vol. 5225, pp. 101-112, 2003.

[9] D. Taillaert, W. Bogaerts, P. Bienstman, T. F. Krauss, P. Van Daele, I. Moerman, S. Verstuyft, K. De Mesel, and R. Baets, "An out-of-plane grating coupler for efficient butt-coupling between compact planar waveguides and single-mode fibers," IEEE J. Quantum Electron., vol. 38, no. 7, pp. 949-955, Jul. 2002. 